\title{
HOMONYMIE DANS LA TERMINILOGIE MILITAIRE FRANCAISE-RUSSE
}

\author{
Dr. Miroslav Stasilo \\ Vilniaus universitetas
}

Sommaire. L'évolution linguistique est caractérisée par des rapports entre les langues. Le russe avait beaucoup de rapports avec la langue française dans le passée, surtout aux XVII-XIX siècles. Il se présente avantageux d'examiner l'apparition des mots français dans la langue russe, en particulier dans le domaine du vocabulaire militaire car l'histoire de France est marquée par de grands dirigeants militaires comme Charlemagne, Napoléon ou Charles de Gaulle. La plupart des termes militaires d'origine française est apparue dans la langue russe à l'époque de Nopléon ler, notamment bataillon, brigadier, général, salut, soldat, etc. Certains mots ont gardé leur signification primaire mais d'autres se sont transformés en recevant parfois une connotation négative, par ex., les vocables ou expressions comme ,sortir", ,cher ami" ou ,cheval“.

Le but principal de ce travail est de soutenir l'idée de l'importance de multilinguisme pour l'évolution linguistique car toutes les langues intéragissent entre elles en devant ainsi plus riches, plus belles et plus accessible aux autres. La comparaison et analyse de l'influence du français sur le russe ainsi que du vocabulaire professionnel des militaires en français et en russe avec la recherche des mots similaires qui ont la même forme orale ou écrite mais possèdent des sens différents ou semblables nous pourra nous aider à répondre à la question s'il y a une origine ou explication unique de l'apparition des homonymes dans le discours militaire français-russe.

Mots-clès: multlinguisme, langue française, langue russe, changement de sens, emprunts, homonymes.

\section{Introduction}

Actualité et problématique. Dans le monde globalisé moderne, il n'y a rien d'inhabituel ni d'exceptionnelle à comprendre ou apprendre une, voire plusieurs langues étrangères. C'est un processus naturel assez fréqent aujourd'hui, surtout dans les pays de l'Europe centrale et orientale comme la Lituanie, la Pologne, la Tchéquie ou la Lettonie, où beaucoup de gens sont au moins bilingues - on y parle deux ou trois langues étrangères, par ex., l'anglais, le russe et le français ou l'allemand. Cela peut être renforcé grâce aux liens établis entre les langues à travers les années et les siècles dont l'homonymie, qui résulte de l'interférence d'une langue 
sur une autre chez les parleurs d'une étendue géographique, culturelle et ethnique bien distincts. Dans notre cas, c'est un rapport assez étroit de la France avec la Russie, entre la culture française et la culture russe dans le passé. La langue française a laissé de profondes traces dans la langue russe, surtout dans la terminologie militaire.

Notre objet de recherche se concentre sur la comparaison et analyse du vocabulaire professionnel des militaires en français et en russe ainsi que la recherche des mots similaires qui ont la même forme orale ou écrite mais possèdent des sens différents ou semblables. Cela pourra nous aider à répondre à la question s'il y a une origine ou explication unique de l'apparition des homonymes dans le discours militaire français-russe. Notre objectif est également de commencer à élaborer une liste basique d'homonymes, utile dans l'apprentissage du français ou du russe langues étrangères ainsi que lors de la traduction du discours militaire français ou russe.

En ce qui concerne les méthodes, nous nous basons sur des théories et concepts de M. Kœssler, J. Derocquigny, O. Akulenko, J. Breuillard, A. Golovnja, K. H. Gottlieb, P. Keruhel, A. Kolganova, M. Seydoux ainsi que des dictionnaires ou encyclopédies liés aux thèmes des emprunts français dans le russe et la présence des homonymes ainsi que des liens entre le français et le russe.

\section{Bilinguisme en Lituanie}

Une majorité de Lituaniens maîtrise au moins une ou deux langues étrangères. En Lituanie, les langues vivantes les plus populaires sont l'anglais, le russe, l'allemand et le français. Voici les données du Département de statique de la République de Lituanie ${ }^{1}$ (cf. Bibliographie. Sources Internet) pour la période de 2017 à 2018 :

Tableau $n^{\circ} 1$. Langues étrangères dans les écoles en Lituanie entre 2017 et 2018

\begin{tabular}{|l|c|c|c|c|c|c|}
\hline & $\begin{array}{c}\text { 1ère langue } \\
\text { étrangère }\end{array}$ & $\mathbf{\%}$ & $\begin{array}{c}\text { 2ème langue } \\
\text { étrangère }\end{array}$ & $\mathbf{\%}$ & $\begin{array}{c}\text { 3ème langue } \\
\text { étrangère }\end{array}$ & $\mathbf{\%}$ \\
\hline Anglais & 287,218 & 98,86 & 1,108 & 0,77 & 0 & 0 \\
\hline Russe & 723 & 0,25 & 114,676 & 78,8 & 490 & 27,54 \\
\hline Allemand & 1,929 & 0,67 & 21,999 & 15,1 & 368 & 20,69 \\
\hline Français & 681 & 0,23 & 7,017 & 4,8 & 412 & 23,16 \\
\hline Autre & 0 & 0 & 745 & 0,51 & 509 & 28,61 \\
\hline
\end{tabular}

Comme on le voit (cf. Tableau ${ }^{\circ}$ 1), la langue choisie par les élèves le plus souvent est l'anglais, ensuite - le russe, l'allemand et finalement le français. Le statut de l'anglais et du russe augmente chaque année, tandis que celui du français et de l'allemand diminue en devenant soit la troisième soit la quatrième langue étrangère. C'est ainsi que la population lituanienne peut être considérée comme une 
nation bilingue ou multilingue.

Le niveau du multilinguisme augmente en Lituanie. Le dernier recensement de la population lituanienne a démontré que le pourcentage des habitants maîtrisant deux langues avait augmenté de 3,9 pourcents : $29 \%$ en 2011 et $25,1 \%$ en 2001 (cf. Graphique $n^{\circ} 1$ ), trois langues - de 1,6 pourcents : $6,6 \%$ en 2011 et $5 \%$ en 2001 , quatre ou plus de langues - de $0,5 \%: 1,3 \%$ en 2011 et $0,8 \%$ en 2001 sur l'échelle générale où 78,6 pourcents maîtrisent au moins une langue étrangère (ibidem) :

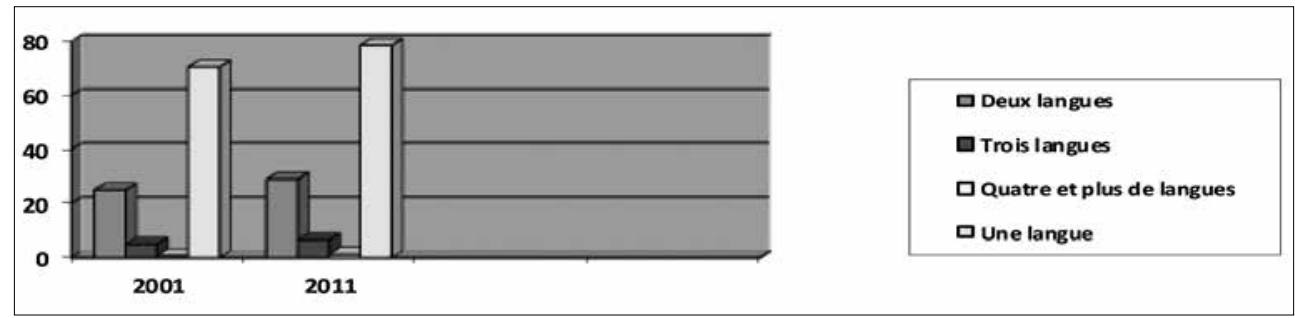

Graphique $n^{\circ} 1$. Maîtrise des langues en Lituanie

Les données d'Eurobaromètre sont encore plus encourageantes concernant le plurilinguisme en Lituanie : selon les enquêtes menées en $2012^{2}$ (cf. Bibliographie. Sources Internet), la population de Lituanie est plus compétente au niveau linguistique que la moyenne de l'UE. D'après ce sondage, ce n'est que 8 pourcents de Lituaniens qui ne parlent aucune langue étrangère, alors qu'en Europe, c'est 46 pourcents ; 92 pourcents parlent au moins une langue étrangère et $54 \%$ en Europe, deux langues - $52 \%$ en Lituanie et $25 \%$ dans l'UE, trois langues $-18 \%$ et $10 \%$ (ibidem).

Les données d'Eurobaromètre démontrent l'importance de l'allemand : 28 pourcents de Lituaniens considèrent la langue allemande la deuxième langue étrangère après l'anglais (ibidem). Pourtant, les données statistiques et la vraie situation de l'apprentissage des langues vivantes en Lituanie ne sont pas les mêmes - entre 2010 et 2011, cette langue s'est retrouvée en 4ème position après l'anglais, le russe et le français (ibidem). A l'Académie, par ex., elle n'a pas été enseignée entre 2013 et 2015 en raison du manque d'étudiants désirant l'apprendre. La même situation est avec le français dont la présence dans les écoles et universités lituaniennes baisse aussi. Cette langue est bien présente sur l'arène de la politique internationale (langue officielle de toutes les plus grandes organisations internationales), riche historiquement et culturellement (l'histoire, la musique, le cinéma, le théâtre, l'architecture etc. Made in France ont une réputation et connotation mondiale) ainsi que scientifiquement (selon plusieurs rapports statistiques, la France se trouve parmi cinque pays qui publient le plus d'articles scientifiques ayant un nombre important de lauréats des prix Nobel). 


\section{Rapports entre la culture française et la culture russe}

La langue russe a plus de rapports avec la langue française que la langue lituanienne, grâce à ses rapports assez intenses dans le passé. Ces liens datent déjà de l'époque du Moyen Age. Par exemple, la fille du souverain de l'âge d'or de La Russie de Kiev Iaroslav le Sage Anne de Kiev s'est mariée avec le roi de France Henri 1er de la famille de Capétiens. Un autre exemple est celui du début du XVIe siècle, quand le Grand-Duc de Moscou Vassili III Ivanovitch a envoyé des lettres au roi français François Ier en recherchant un appui politique en Europe et en essayant d'établir des relations diplomatiques entre la Russie et la France. Ces lettres étaient malheureusement interceptées par les Polonais et il fallait attendre jusqu'à l'année 1668, quand le gouverneur célèbre de Borovsk Petr Ivanovic Potemkin a été chaleureusement accueilli par le roi de France Louis XIV (Seydoux, 1968: 236). C'est à partir de cette date-là qu'on peut parler du début des relations diplomatiques périodiques entre les deux pays.

Jusqu'au milieu du XVIIe siècle, la Russie était très peu connue en France. De même, le français et la culture française n'avaient pas la même influence sur la langue et la culture russe que celle qu'on y aperçoit à partir du règne de Pierre Le Grand (1682-1725), quand tout l'Etat russe s'est dirigé brusquement vers l'Europe. Les deux pays commencent à s'intéresser l'un à l'autre grâce à leurs conquêtes : la Russie gagne contre les alliés français tels que la Suède, la Pologne ou la Turquie alors que la présence de la culture française était incontestable dans tous les grandes cours européennes à l'époque. D'ailleurs, les enfants de Pierre Ier, dont la future impératrice de Russie Elisabeth Ière (ou Elizaveta Petrovna), ont été entourés et élevés par des enseignants français, qui leur ont injecté une très bonne connassance de la langue française avec une grande sympathie pour la France. Cela a ammené petit à petit à la " gallomanie » (Breuillard / Keruhel, 1979:468), c'est à dire des mots français employés en russe.

La culture et la langue françaises se sont bien enracinées en Russie pendant la gouvernance de l'impératrice Elizaveta Petrovna (1741-1762) à tel point que les mots français, employés souvent par des aristocrates ou bien des diplomates, entrent dans la langue russe de tous les jours, surtout dans les domaines d'archtecture, de politique, d'art militaire et d'administration, par ex., « ансамбль, тротуар, ассоциаиия » (Gerber, 2018). De plus, la langue française devient de plus en plus présente dans les établissements d'enseignement supérieur où certaines matières n'étaient enseignées qu'en français comme la physique ou les sciences politiques, par exemple. Sans oublier bien sûr l'arrivée de Jean-Baptiste Landé, invité par le conseiller d'Anne de Russie Pietro Mira, en 1734. C'est ce Français qui a décidé de fonder une grande école de danse en 1738, devenue ensuite l'École théâtrale impériale de Saint-Pétersbourg (1783), connue actuellement dans le monde entier sous le nom de l'Académie de ballet Vaganova. Et c'est aussi grâce à cette école que tous les termes de ballet classique sont d'origine française maintenant : adage (mouve- 
ment lent), battement (lancement de la jambe en avant), chassé (un pas simple), demi-plié (pliage des jambes sans soulever les talons), etc.

L'impératrice russe Catherine La Grande (ou Catherine II de Russie) continue le mouvement de la gallomanie dans la langue russe en y faisant entrer des vocables appartenant aux domaines de littérature, de théâtre et de philosophie. Les mots comme « интерьер (intérieur), люстра (lustre), канделябр (candélabre), абажур (abat-jour) » (ibid.) datent de l'époque de Catherine II de Russie (17621796), qui était une amie épistolaire avec Voltaire, Denis Diderot et Jean le Rond d'Alembert. C'est aussi elle qui a fondé l'Académie des beaux-arts, dont un grand nombre de professeurs était très proche au milieu parsisien et dont le programme d'enseignement reste actuel de nos jours, en 1757 à Saint-Pétersbourg. Catherine La Grande voulait également que les intellectuels russes voient Voltaire et Molière sous l'interprétation de la Comédie française. Au moment où la troupe de la Comédie française a montré son spectacle à Saint-Pétersbourg en 1770, la culture française a encore une fois ébloui la haute société russe (Zaborov, 1968). C'est aussi la naissance des traditions théâtrales de l'école russe pour laquelle la langue française tiennent à coeur même aujourd'hui dont la preuve est la présence de l'enseignement du français dans les plus grandes écoles théatrales de Moscou, par ex., École-studio MKhAT (en russe : Школа-студия МХАT) ou GITIS.

Comme on l'a vu ci-dessus, la période entre les XVIIe et XVIIIe siècles était très favorable pour toute la culture française. Beaucoup de nobles riches russes voulaient avoir un chef de cuisine originaire de France. Cela a conduit à l'apparition de nouvelles appellations des plats d'origine française, par ex., cyфлe (soufflé) - gâteau avec de la crème à lintérieur, шампиньон (champignon) - type particulier de champignons, салат (salade) - salades, etc. Les gens nobles et riches invitaient souvent des gens d'origine française en Russie pour qu'ils enseignent à leurs enfants. C'est ainsi que la langue française était parfois mieux maîtrisée que la langue maternelle russe. La connaissance du français est devenue par conséquent incontournable pour une personne civilisée et éduquée. Beaucoup de gens célèbres communiquaient et écrivaient en français en Russie aux XVIIIe - XIXe siècles : Grigori Alexandrovitch Potemkin, les Orlov, les Dolgorouki, les Bestoujev, les Galitzine, les Schouvalov, les Tolstoï, les Mentchikov, les frères Panine, etc.

La place de la langue française dans la culture russe a été renforcée grâce à l'importance de la période de XVIIIe-XIXe siècles, considérée comme un grand moment de mutations et d'ouverture vers l'Occident en Russie par plusieurs chercheurs et intellectuels (Mézin, Raeuckij, 2002; Gretchanaia, Viollet, 2011). De nouveaux processus et sphères, de nouvelles choses et objets, qui $n^{\prime} y$ existaient pas auparavant, sont originaires de la culture et de la langue française. L'un des résultats d'une telle évolution de la société russe est l'apparition de la terminologie française dont beaucoup de termes sont utilisés à ce jour en russe (Sinicyn, 2018). Or, ce n'est pas si simple de distinguer si le vocable, qui semble d'origine française, est vraiment emprunter directement du français (Breuillard / Keruhel, 1979 : 468-469). 


\section{Changement de sens}

La plupart des mots russes d'origine française date de la période historique du XVIIIe au XIXe siècle. La langue française a évolué depuis ce temps-là et beaucoup de ces mots d'origine française sont devenus des archaismes : soit disparus de l'usage quotidien, soit très peu employés, soit ils ont changé de sens. De plus, parfois, le processus de gallomanie était si avale en russe (et en d'autres langues aussi, d'ailleurs) qu'on oubliait ou transformait la signification initiale lexicale, par ex., le mot français « anecdote », qui signifie en français « Bref récit d'un fait curieux ou pittoresque, susceptible de divertir ; histoire. » (LAROUSSE), n'a été accepté par la langue russe que comme une petite histoire drôle, fréquemment vulgaire et créée par des anonymes.

" Pourquoi les mots d'origine étrangère changent parfois de sens ? ", « Pourquoi certains emprunts disparaissent et d'autres restent ? », « Comment et pourquoi les homonymes apparassent-ils? ». Pour répondre à ces questions ainsi qu'afin de comprendre les homonymes français et russes en évaluant leur signification, on rappelle le schéma de Pierre Martel, initié par la théorie structuraliste de Férdinand de Saussure, concernant le changement de sens dans la langue française au Canada :

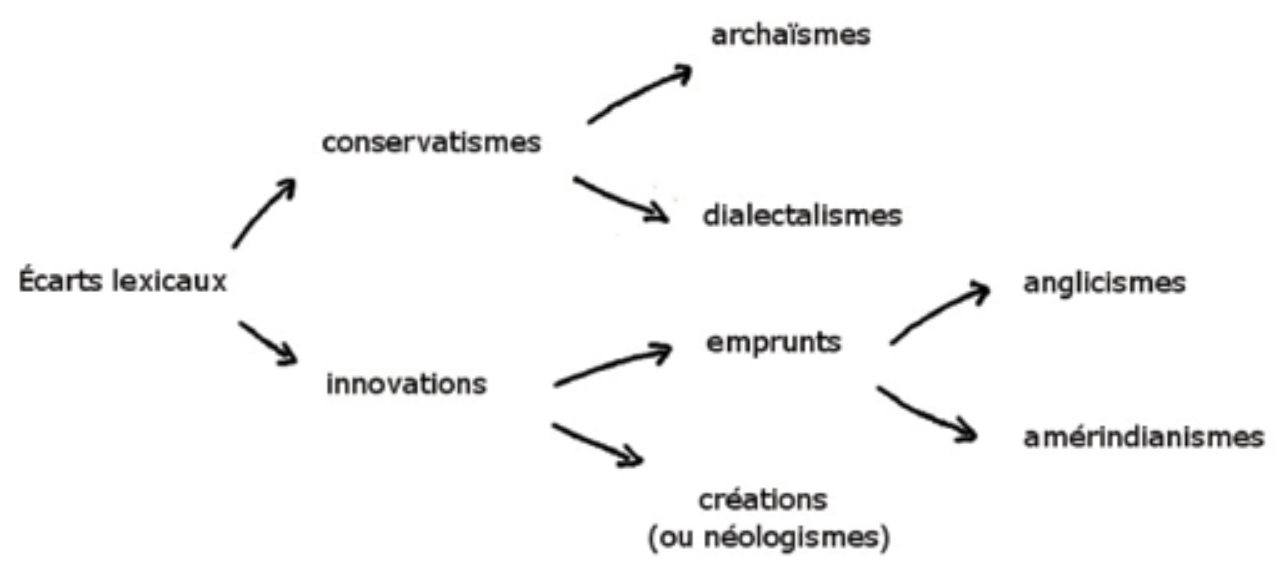

Graphique $n^{\circ} 2$. Signification lexicale ${ }^{3}$

La graphique (cf. ci-dessus) représente deux grands types de changements linguistiques : conservatismes et innovations. Le premier type englobe les archaismes et dialectismes alors que le deuxième - les emprunts et néologismes. Les homonymes, qui sont au centre de nos recherches, ce sont des mots du lexique moderne. Ils se prononcent ou s'écrivent de la même manière mais n'ont pas le même sens. C'est pourquoi nous nous focalisons sur le deuxième groupe de changements linguistiques, c.-à.-d., innovations. 


\section{Exemples des emprunts et des homonymes dans la terminologie militaire}

russe

Tous les exemples qui suivent appartiennent au moment de la guerre de Napoléon contre la Russie. C'est une période contradictoire : d'un côté, les Russes se battaient contre l'invasion de l'impérateur français ; d'un autre côté, ils admiraient la France avec sa langue et culture. Le roman de Lev (ou Léon) Tolstoï « La Guerre et la Paix » illustre merveilleusement toute la complexité de cette situation historique.

Le premier terme, appartenant au discours militaire français-russe, est un ordre, qu'on peut entendre assez souvent aujourd'hui en provenance d'une mère s'adressant à son enfant : « Mapu! ». Ce n'est pas un homonyme même si sa prononciation est identique à l'impératif du verbe français « marcher » à la deuxième personne du singulier car son emploi en russe est porche à celui en français. Les Russes pouvaient entendre cela au moment quand les commandants de l'armée napoléonienne criaient à leurs soldats : «En avant, marche! ». Max Vasmer confirme les racines françaises de cette expression populaire sans avoir précisé cependant son contexte, ni le moment d'apparition en russe (cf. Dictionnaire etymologique de Vasmer).

Un autre exemple est un homonyme car son emploi n'est pas le même qu'en français. Ce mot provient également d'un verbe. C'est le mot «copmup» qui se prononce de la même manière que l'infinitif du verbe de deuxième groupe « sortir ». D'après une version, ce vocable est probablement apparu à la fin du XVIIe ou au début du XVIIIe siècle dans le milieu d'aristocrates. Quand les princes et les princesses voulurent se mettre en selle, ils se dirigèrent vers les Français en disant : «Je dois sortir ». Ainsi, le mot « copmup », qui signifie actuellement « toilettes » dans la langue parlée, est une sorte d'euphémisme. D'après une autre version, ce vocable est apparu en 1812, lorsque les prisonniers français en Russie ont été invités à " sortir ». Les soldats russes ont alors interprété ces syllabes à leur propre manière.

Un autre cas homonymique représente une situation assez paradoxale car son emploi positif dans la langue-source (langue $A$ ) a reçu une connotation négative dans la lanague $B$. Le mot «шаромыга» signifie « trompeur, scélérat, mendiant ». D'après l'hypothèse la plus répandue, les soldats de Napoléon, qui étaient otages, s'adressait aux Russes avec 1'expression «cher ami », lorsqu'ils suppliaient les Russes en demandant grâce et de les nourrir. On peut imaginer alors que la valeur négative de cet appel sincère en français venait de l'indulgence russe envers le soldat français vaincu se rtrouvant dans une situation regrettable et pitoyable.

Parfois, les mots ou expressions perdent leur côté positif, comme on l'a vu ci-dessus. Cela est souvent dû au contexte négatif de l'incorporation d'un mot d'une autre langue. C'était la guerre entre la Russie et la France. Les Français, même s'ils avaient une image favorable en Russie, étaient considérés comme des envaisseurs, occupants et ennemis. Tout au long de la campagne militaire de 1812, par exemple, 
les Russes se retiraient devant 1 'armée de Napoléon ayant adopté une politique de la terre brûlée ainsi que de la destruction d'objets matériels, d'approvisionnements et de récoltes en cours de route.

Un autre exemple d'une transformation négative pourrait être le vocable français « cheval » que les Russses entendaient souvent parlé par les soldats et officiers français. D'après une hypothèse, après la perte de Napoléon dans la bataille de la Bérézina (ou de Borisov), l'impérateur français avait laissé derrière lui un grand nombre de soldats et de chevaux morts. Ceux, qui se retiraient et étaient encore vivants, cherchaient des cadavres d'animaux et criaient " cheval, cheval» dès qu'ils en retrouvaient quelques-uns. Donc, le paysan russe, ayant entendu une telle exclamation répétée, associait ce son à une image négative, puante et confuse. Finalement, le mot français a été emprunté par les Russes. Ce mot signifie maintenant une personne illetrée, peu développée, ignare. On emploit le vocable « шваль », si on veut humilier qulequ'un en russe. Selon une autre version, le mot vient directement de l'insulte dans les jeux de cartes où la figure d'un chevalier n'était pas une bonne carte. Et d'après d'autres sources, ce mot vient de «Schwall », qui signifie une masse gonflée en allemand. Selon l'hypothèse de Vasmer (cf. Dictionnaire etymologique de Vasmer), la racine de cette injure est proprement slave car elle provient du mot propre d'Ivachka Cheval (Ивашка Шваль). C'était un homme concret - un tailleur qui, lors de l'assaut de Novgorod par les Suédois en 1614, a ouvert les portes de la ville aux ennemies en échange d'un pot-de-vin, leur assurant ainsi la victoire. C'est pourquoi, selon Vasmer ce mot a une connotation négative.

Le dernier exemple d'un terme militaire dont l'origine provoque également des discussions. C'est le mot « brigadier ». En français, ce mot existe depuis le XVIIe siècle. Il est dérivé du mot « brigade » et signifiait auparavant le suivant : « officier dont le grade tenait le milieu entre ceux du colonel et du maréchal de camp; officier supérieur dans certaines armées » (Dictionnaire de 1‘Académie Française). Aujourd'hui, les Français emploient ce terme pour appeler un « militaire pourvu du grade le moins élevé dans la cavalerie, les blindés, l'artillerie, le train et la gendarmerie, équivalant au grade de caporal dans l'infanterie et le génie [...] chef d'un groupe d'ouvriers ou d'employés » (ibidem).

Le mot russe « бригадир » est apparu au début du XVIIIe siècle et s'est répandu grâce à l'armée napoléonienne. En russe, il désigne « un chef d'équipe d'ouvriers ». Selon le dictionnaire étymologique russe, son origine est française mais sa prononciation et son emploi étaient influencés par le mot allemand « der Brigadier ». Le sens de ce mot correspond par analogie au vocable français en gardant une seule signification, qui existe en russe de nos jours. Donc, le « brigadier » français est interprété dans la langue russe par rapport à sa ressemblance, c'est à dire métaphoriquement. Les Russes ont associé par conséquent deux choses qui leur semblaient similaires.

Pour finir le sujet des emprunts et homonymes, issus de la campagne de Russie de 1812, il faut dire que les Russes ont glorifié le nom de Napoléon et sa pré- 
sence dans le pays via un gâteau très populaire, appelé « торт Наполеон » (gâteau Napoléon). C'est un lointain " cousin » du mille-feuilles dont la texture est assez semblable à celle de " Napoléon », appelé en France comme " un gâteau russe ». On dirait, ce gâteau est un exemple de l'homonymie culinaire. Mais c'est un autre sujet de travail.

\section{Conclusions}

Pour conclure, on peut dire que les traces de la langue française sont bien présentes en russe contemporain. La langue évolue grâce aux contacts avec d'autres langues et cultures. C'est surtout grâce à l'influence du français et de la France aux XVII-XIX siècles, quand l'Etat de Russie était en train de se former en s'ouvrant vers l'Occident, que la langue russe s'est modernisée en devenant mieux organisée et structurée car la langue française l'était déjà à l'époque. Avant cette période, le russe écrit était plutôt employé dans des textes religieux anciens. Après la rencontre du français avec la langue russe, l'élite et les intellectuels russes ont commencé à s'intéresser à l'évolution de leur langue littéraire dont la preuve est l'émergence de grands poètes et écrivains comme Léon Tolstoï, Fiodor Dostoïevski ou Alexandre Pouchkine.

Quant à la réponse concernant la question s'il y a une origine ou explication unique de l'apparition des homonymes dans le discours militaire français-russe, il nous paraît essentiel de souligner l'importance des relations économiques, politiques et culturelles entre les pays et les langues. C'est ainsi que la campagne de Russie de 1812 a laissé plusieurs termes militaires d'origine française dans la langue russe. La signification positive de ces termes recevait parfois une connotation négative suite à l'influence d'un contexte historique défavorable. Pour finir, nous avons vu que le niveau de bilinguisme augmente généralement en Lituanie. On espère que le nombre de gens maîtrisant plus d'une ou de deux langues étrangères va également augmenter grâce à la compréhension des relations entre les langues, dans notre cas - entre le russe et le français.

\section{Bibliographie}

1. Breuillard P./ Keruhel J. (1979). L'identification des emprunts français dans le russe du début du XIXe siècle. Revue des études slaves 52. Paris.

2. Gerber J. (2018). Приключения франиузских слов в России. Consulté le 15 avril 2020 sur: https://chosesnormales.wordpress.com/2018/06/18/1491/

3. Gretchanaia E., Viollet C. (2011). Diaristes et épistolières russes (fin XVIIIe - début XIXe siècle) : reflets de l'histoire. Revue de l'Association Mnémosyne « Genre et Histoire » [en ligne], 9. Automne 2011. Consulté le 2 mai 2020 sur : https://journals.openedition.org/genrehistoire/1396

4. Mézin A., Raeuckij V. S. (2002). Projet d'un Dictionnaire des Français en 
Russie au XVIIIe siècle. Cahiers du monde russe [en ligne], 43/2-3. Cosulté le 23 avril 2020 sur: https://journals.openedition.org/monderusse/8498

5. Saussure F. (2002). Écrits de linguistique générale. Gallimard. Paris.

6. Seydoux M. (1968). Les ambassades russes à la cour de Louis XIV. La revue Cahiers du monde russe, vol. 9. Paris.

7. Sinicyn, S. (2018). Французский язык и культура в российской жизни в XVIII-XIX веках. Consulté le 4 mai 2020 sur: https://www.le-francais.ru/cours/ chapitre $4 /$

8. Zaborov, P. (1968). Le théâtre de Voltaire en Russie au XVIIIe siècle. $C a$ hiers du monde russe, vol. 9. Paris.

\section{Dictionnaires :}

1. Dictionnaire de l'Académie française. Consulté le 7 mai 2020 sur : https:// www.dictionnaire-academie.fr/article/A9B2136

2. Dictionnaire etymologique de Shaposhnikov [Этимологический словарь Шапошникова] (2010). Consulté le 30 avril 2020 sur: http://www.slovorod.ru/ etym-shaposhnikov/shap-b.htm

3. Dictionnaire etymologique de Vasmer [Этимологический словарь русского языка (М.Р. Фасмер)] (2019). Consulté le 29 avril 2020 sur : http://rusyaz.niv.ru: http://rus-yaz.niv.ru/doc/etymological-dictionary/fc/slovar-203-4.htm\#zag-6891

4. LAROUSSE (2018). Consulté le 15 avril 2020 sur: https://www.larousse. fr/dictionnaires/francais-monolingue

\section{Sources Internet:}

1 https://osp.stat.gov.lt/documents/10180/217110/Gyv+pagal+išsilavinima _ir_kalbu_mokejima.pdf/b75c5e7a-e733-48fa-95e4-83d485c6726a (Consulté le 10 avril 2020)

2 http://www.veidas.lt/tag/kalbos (Consulté le 12 avril 2020)

3 http://www.linguistes.com/mots/variation.html (Consulté le 17 avril 2020) 


\title{
HOMONYMY IN THE FRENCH-RUSSIAN MILITARY TERMINOLOGY
}

\author{
Dr. Miroslav Stasilo
}

Vilnius University

\section{Summary}

Linguistic evolution is characterized by relationships among languages. Russian had a lot to do with the French language in the past, especially in the 17th-19th centuries. It is advantageous to examine the emergence of French words in the Russian language, especially in the field of military vocabulary, since the history of France is marked by great military rulers such as Charlemagne, Napoleon or Charles de Gaulle. Most of the military terms of French origin appeared in the Russian language during the time of Napoleon I, including battalion (French: bataillon), brigadier (French: brigadier), general (French: général), salute (French: salut), soldier (French: soldat), etc. Some words have kept their primary meanings but others have been transformed, sometimes receiving a negative connotation, e.g. the words or expressions like go out (French: sortir), dear friend (French: cher ami) or horse (French: cheval).

The main goal of this work is to support the idea of the importance of multilingualism for linguistic evolution because all languages interact with each other making them richer, more beautiful and more accessible to others. The comparison and analysis of the influence of French on Russian as well as the professional vocabulary of the military in French and Russian with the search for similar words which have the same oral or written form but have different or similar meanings can help us to answer the question whether there is a single origin or explanation for the appearance of homonyms in the French-Russian military discourse.

Keywords: multilinguism, French language, Russian language, change of meaning, borrowings, homonyms. 


\section{AUTORIAUS LYDRAŠTIS}

Autoriaus vardas, pavardè: Miroslav Stasilo

Mokslo laipsnis ir vardas: filologijos mokslų daktaras

Darbo vieta ir pareigos: Generolo Jono Žemaičio Lietuvos karo akademijos Užsienio kalbų katedra; Vilniaus universiteto Filologijos fakulteto Taikomosios kalbotyros institutas

Autoriaus mokslinių interesų sritys: užsienio kalbos, sociolingvistika, politikos mokslai

Telefonas ir el. pašto adresas: + 37061144811 ;

francaisprancuzas@yahoo.fr

\section{AUTHOR'S COVER LETTER}

Author's name and surname: Miroslav Stasilo

Academic degree and name: Doctor of Linguistics

Workplace and position: General Jonas Žemaitis Military Academy of Lithuania, Department of Foreign Languages; Vilnius University, Faculty of Philology, Applied Linguistics Institute

Author's research interests: foreign language, sociolinguistics, political science

Telephone and e-mail address: +37061144 811;

francaisprancuzas@yahoo.fr 